\title{
Artículo
}

\section{Ejercicio en Enfermedad Renal Crónica}

\section{Exercise in chronic renal disease}

\author{
Autoras: \\ Dra. Vanessa Uclés Villalobos \\ Especialista Medicina Física y Rehabilitación, HCG. \\ Dra. Celina Vargas Arguedas \\ Especialista Medicina Física y Rehabilitación, Servicio Social en HNN.
}

Correspondencia a: dravanessaucles@gmail.com

Abreviaturas:

ERC: Enfermedad renal crónica

TFG: Tasa de filtración glomerular

HTA: Hipertensión arterial

DM: Diabetes mellitus
AM: Adulto mayor

AVD: Actividades de Vida Diaria

HD: Hemodiálisis

\section{Descriptores:}

Ejercicio físico/Exercise

Insuficiencia renal crónica/ Chronic Renal Insufficiency

Diálisis renal/Renal Dialysis

Trasplante de riñón/Renal transplantation

\section{Generalidades}

La Enfermedad renal crónica (ERC) se define como la presencia de daño renal o la reducción de Tasa de filtración glomerular (TFG) $<60 \mathrm{ml} /$ $\min / 1,73 \mathrm{~m} 2$ por 3 meses o más. 
Existen 5 estadíos:

\begin{tabular}{lll} 
Estadio & Descripción & FG $\left(\mathbf{m l} / \mathbf{m i n} / \mathbf{1 , 7 3} \mathbf{~ m}^{2}\right)$ \\
-- & Riesgo aumentado de ERC & 60 con factores de riesgo* \\
\hline 1 & Daño renal + con FG normal & 90 \\
\hline 2 & Daño renal + con FG ligeramente disminuido & $60-89$ \\
\hline 3 & FG moderadamente disminuido & $30-59$ \\
\hline 4 & FG gravemente disminuido & $15-29$ \\
\hline 5 & Fallo renal & $<15$ o diálisis
\end{tabular}

Se considera que en el estadio 1 el porcentaje de función del riñón está en 90-100\%, 60-89\% en el estadio 2, 30-59\% estadio 3, 15-29\% estadio 4 y $<15 \%$ en estadio 5. El estadio 3 se divide en 3 a y $3 \mathrm{~b}$, con porcentajes de $45 / 59 \%$ y $30-44 \%$ respectivamente.

Los estadios permiten planificar el tratamiento: en el 1 se observa y controla HTA, en el 2 y 3 se observa y controla HTA y factores de riesgo y en el 4 se planea el fallo renal, por lo tanto, la diálisis.

Existen varios factores predictores de ERC secundaria. La edad, HTA y DM son clave, ya que hay una tendencia al envejecimiento global de la población, y porque la HTA y la DM son un problema de salud pública global que sigue en aumento.

La TFG disminuye conforme avanza la edad, por lo que ERC aumenta su prevalencia en AM, y en esta población la ERC se asocia con el desarrollo de discapacidad y predice que gran parte de la población requerirá de asistencia y de cuidados específicos. Con una TFG<

$45 \mathrm{ml} / \mathrm{min}$, los AM tienen tres veces más probabilidad de deterioro de sus actividades de la vida diaria (AVD), y tienen mayor declive en la movilidad, lo que lleva a una disminución de la capacidad para salir de su casa, barrio o para involucrarse en actividades sociales y participación (familia, iglesia) (1).
Además, la ERC se asocia con mayor riesgo de limitación en las habilidades y capacidades para las AVD, independientemente de edad, género, comorbilidades y eventos cardiovasculares, tanto en pacientes en estadíos finales, como en pacientes con estadíos iniciales (TFG promedio $60 \mathrm{ml} / \mathrm{min} / 1.73 \mathrm{~m} 2$ ) (2).

Por otra parte, el bajo nivel de actividad física y el pobre funcionamiento físico se asocian fuertemente a mortalidad y a malos resultados clínicos en estos pacientes, independientemente del tratamiento que tengan (1).

Actualmente la prevalencia es de $10-16 \%$ en adultos a nivel mundial y en mayores de 64 años va de 23,4 a 35,8\% (1). En Costa Rica, el número de pacientes con diagnóstico de ERC se triplicó entre 2011 a 2018, con más de 800 casos en este último año. De estos casos, 208 pacientes recibían tratamiento de hemodiálisis (HD) y 647 estaban en diálisis peritoneal crónica ambulatoria (DPCA).

La prevalencia de ERC definitivamente aumenta con la edad: $31-45 \%$ de las personas mayores de 70 años tienen un estadio $3^{\mathrm{a}}$ o peor, lo que aumenta la mortalidad (3).

El costo de tratar pacientes con ERC en estadios finales representa un problema de salud pública: en Europa menos del $0.1 \%$ de la 
población necesita terapia de diálisis y eso representa el $2 \%$ del presupuesto para atención en salud (1).

Cabe resaltar que si bien la diálisis conduce a una mejoría de los síntomas y de la sobrevida, no cura la enfermedad, y tiene morbimortalidad asociada, especialmente a nivel cardiovascular, y afecta la calidad de vida. Se hace necesario entonces buscar intervenciones complementarias a la diálisis.

La enfermedad cardiovacular es la mayor fuente de morbi-mortalidad en pacientes con ERC, 2 veces más que la población sin ERC (82.1\% vrs $45.2 \%)(4)$.

\section{Evidencia del ejercicio en la ERC}

Estudios epidemiológicos indican que inactividad física es un factor de riesgo independiente

para el desarrollo y progresión de ERC y sus complicaciones asociadas. En estudios donde se controlan las enfermedades crónicas más prevalentes (DM, HTA y enfermedad cardiovascular), las personas con menor nivel de actividad física son 10 veces más propensas a desarrollar ERC (5). De hecho, se ha visto que el sedentarismo por si solo es un factor de riesgo de mortalidad tan importante como la hipoalbuminemia en pacientes con diálisis peritoneal y hemodiálisis (6).

Los pacientes con ERC tienen una alta prevalencia de enfermedad cardiovascular asociada con o exacerbada por la inactividad (4).

Por su parte, existe evidencia clara sobre los beneficios del ejercicio regular en el acondicionamiento físico, en la capacidad de marcha, en parámetros cardiovasculares como PA y FC, en la calidad de vida relacionada a salud y en parámetros nutricionales (como albúmina, prealbúmina e ingesta energética) en adultos con ERC, independientemente del estadio en que se encuentren, si están recibiendo diálisis (hemodiálisis o diálisis peritoneal) o si ya fueron trasplantados (7) (8).
En efecto, los estudios han demostrado que mayores niveles de actividad física se asocian a reducción de $37 \%$ de la tasa de pérdida de función renal en personas mayores de 65 años con ERC 2-3. También se ha visto que un $\mathrm{VO} 2$ pico $>17.5 \mathrm{ml} / \mathrm{kg} / \mathrm{min}$ se asocia con mejores tasas de supervivencia de más de 3,5 años en pacientes en hemodiálisis, así como que una velocidad de marcha $>1,3 \mathrm{~m} / \mathrm{s}$ se asocia con 18-26\% de reducción de riesgo de deterioro de función renal en ERC 2-4. Del puntaje del cuestionario SF-36 sobre función física reportado por los pacientes, un aumento de 1 punto corresponde a una reducción del $2 \%$ en tasa de mortalidad, mientras que una puntuación por debajo de 25 se asocia con $93 \%$ de aumento en el riesgo de muerte y $56 \%$ de aumento en el riesgo de hospitalización en pacientes con diálisis (9) (5) (10).

El pico de VO2 es uno de los parámetros más estudiados de la función cardiopulmonar. Un metaanálisis del 2019 que valora los efectos del ejercicio aeróbico en pacientes con ERC demostró que este tipo de ejercicio mejora el VO2 de forma significativa todo en pacientes en diálisis, así como los niveles de HDL-C y la calidad de vida (11). Otro metaanálisis del 2014 de 24 ensayos clínicos controlados demostró que el ejercicio intradialítico mejora la eficacia de la diálisis, el consumo máximo de oxígeno (pico de VO2) y el desempeño en la caminata de 6 minutos (12). La mejora en la eficacia de la diálisis se puede explicar por el aumento del flujo sanguíneo que se da durante el ejercicio en los músculos, lo cual aumenta la eliminación de tóxicos del dializador (9) (13).

Además, existe una relación entre la rehabilitación renal, que mejora la capacidad de ejercicio, y la reducción de la morbilidad y de la mortalidad. En un estudios longitudinal retrospectivo con 757 pacientes $(242$ en hemodiálisis, 221 con trasplante renal, 43 con diálisis peritoneal y 251 sin diálisis) se valoró la relación entre la rehabilitación renal y 
la ocurrencia de eventos adversos (muerte, accidente cerebrovascular, infarto agudo del miocardio y hospitalizaciones por insuficiencia cardiaca). Los pacientes que lograron completar la rehabilitación renal de 12 semanas mostraron, mostraron una mejoría en los parámetro de la marcha y un menor riesgo de eventos adversos que aquellos que no completaron el programa (14). Adicionalmente, varios estudios han comprobado que el riesgo de mortalidad tiende a disminuir con el aumento de la frecuencia de ejercicio (15).

También se puede citar el estudio DOPPS de Tentori et al, que evidenció que de 20920 pacientes, aquellos que se ejercitaba en la unidad de diálisis al menos 1 vez por semana tenían $37 \%$ menor riesgo de mortalidad por cualquier causa y $27 \%$ menor riesgo de hospitalización por fracturas, comparado con lo que se ejercitan menos de 1 vez a la semana. Además demostró que el ejercicio en la unidad de diálisis se asocia con mejor calidad de vida y funcionamiento físico, mejor calidad de sueño, menor limitación a la actividad por dolor, menor anorexia y menos síntomas depresivos (16).

En general, si bien la evidencia respecto a los afectos y beneficios del ejercicio en pacientes en diálisis es muy amplia y clara, faltan estudios que evidencien estos mismos beneficios en pacientes con ERC pre diálisis. Los estudios existentes hasta el momento sugieren que el ejercicio en este grupo de pacientes confiere protección contra el deterioro de la función cardiaca y el desarrollo de enfermedades cardiovasculares al mejorar los factores de riesgo asociados.

Además existe evidencia de que mejora la capacidad de ejercitarse y las medidas de función física, y el ejercicio de resistencia también puede mejorar en este grupo la fuerza, la masa y la función muscular (17) (8).
A largo plazo, los programas de rehabilitación y ejercicio en pacientes con ERC han demostrado un mayor apego de los pacientes al ejercicio al año, comparado con controles que no participaron en un programa de rehabilitación renal. Además, al año existe una mejoría clínicamente significativa en la función física de base de los pacientes que participaron en el programa de ejercicio comparado con aquellos que no (18).

Respecto al deterioro cognitivo que desarrollan los pacientes con ERC, se dice que puede deberse a enfermedad de pequeño vaso por la disfunción vascular que esta genera (19). Este deterioro, especialmente en las funciones ejecutivas, empeora con el mantenimiento de la terapia de diálisis por retención de toxinas urémicas, isquemia cerebral recurrente e inactividad, que pueden llevar finalmente a demencia y muerte. El ejercicio domiciliario e intradialítico son de las intervenciones no farmacológicas más reconocidas para mantener la función cognitiva en estos pacientes, sumado al entrenamiento cognitivo (20).

Otro beneficio del ejercicio que se ha estudiado en esta población es respecto al síndrome de piernas inquietas, el cual puede afectar hasta un tercio de los pacientes con ERC. En una revisión sistemática del 2014 donde se evaluaron distintas terapias para tratar este síndrome, el ejercicio demostró reducir la gravedad de los síntomas hasta un 58\%, mejorar la calidad de vida asociada a este en $18-25 \%$ y la calidad del sueño de $20-50 \%$ (21).

También se ha visto el efecto positivo del ejercicio en la densidad mineral ósea de los pacientes con ERC, el cual reduce el porcentaje de osteoporosis en los grupos que se ejercitan (22). Considerando que la actividad física es un factor que contribuye a proteger contra la pérdida de masa ósea/osteodistrofia, que se asocia a un riesgo aumentado de fracturas no traumáticas, desórdenes 
musculares y neurológicos, en pacientes con enfermedad renal, se refuerza la necesidad de incluir la prescripción de ejercicio en el programa de rehabilitación para mejorar el pronóstico (23).

Los clínicos deben estar alertas de la importancia de estrategias de rehabilitación para disminuir la instauración y progresión de la osteoartrosis de rodilla en pacientes con falla renal, ya que el cartílago femoral es más delgado een personas con falla renal crónica que en los controles sanos, por lo tanto, con alto riesgo de desarrollarla.

Sobre el hiperparatiroidismo secundario, un estudio transversal demostró que los niveles de hormona paratiroidea libre tiene una relación inversa con la actividad física diaria, y esto es importante pues esta condición aumenta la mortalidad de los pacientes que la padecen y empeora su calidad de vida (24).

Así, es evidente que se requieren programas bien estructurados de prevención primaria que busquen reducir los factores de riesgo para ERC, asociados a estrategias de rehabilitación e intervenciones apropiadas durante la enfermedad renal para reducir el impacto que tendrá la ERC en la aparición de discapacidad y sus costos asociados. El proceso de rehabilitación aborda al paciente desde una visión biopsicosocial, con la meta de disminuir la carga de la discapacidad y aumentar las oportunidades para lograr la mayor autonomía posible. Es un proceso centrado en el paciente y cada proceso de rehabilitación debe individualizarse considerando el estadío de la enfermedad, las complicaciones y las comorbilidades, así como la funcionalidad previa y la habilidad residual. El equipo en rehabilitación debe ser interdisciplinario, formado por distintos profesionales en fisiatría, geriatría, nefrología, enfermería, trabajo social, terapia ocupaciones y terapia física (1).
Una encuesta que se llevó a cabo en Europa, Reino Unido y EUA intentó documentar las prácticas y actitudes de nefrólogos y de equipos multidisciplinarios acerca del consejo de ejercicio en estos pacientes. La encuesta reportó que, aunque el $100 \%$ sabía los beneficios del ejercicio, menos del 50\% lo recomendaba y menos del $25 \%$ facilitaba el acceso a programas de ejercicio por medio de información escrita o por medio de una referencia a un grupo clínico o comunitario (4). Esto expone la necesidad que existe en que los servicios para pacientes con ERC deben evolucionar al incorporar opciones de rehabilitación con ejercicio, como parte del cuidado integral que esta población amerita.

Por ejemplo, la rehabilitación cardiaca en pacientes con ERC mejora la capacidad para el ejercicio y calidad de vida general y trae varios efectos beneficiosos en los factores de riesgo para enfermedad cariovascular, disminuye significativamente eventos coronarios mayores, mortalidad cardiaca y todas las causas de muerte (4) (9) (10).

En resumen, el ejercicio aeróbico disminuye microalbuminuria, protege del estrés oxidativo, puede aumentar la tasa de filtración glomerular, mejora escalas de síntomas, perfiles de impacto de la enfermedad y calidad de vida. El ejercicio de resistencia disminuye la inflamación, aumnta la albúmina sérica, mantiene el peso corporal, aumenta la fuerza muscular y tasa de filtración glomerular en el paciente con ERC sin hemodiálisis. Y en hemodiálisis también aumenta la sensibilidad a la insulina, hemoglobina y fuerza muscular, mejora perfil lipídico y calidad de vida y disminuye la presión arterial si es aeróbico. Aumenta la fuerza muscular y funcionalidad física si es de resistencia. $\mathrm{Y}$ combinados mejora la fuerza muscular, fitness cardiaco, capacidad de trabajo y posibilidad de adecuación de diálisis (25). 
Entonces el entrenamiento físico se recomienda como modalidad terapéutica complementria por 3 métodos:

- Programa en consulta externa supervisado en un centro de rehabilitación (servicio)

- Programa de ejercicio en casa

- Programa de ejercicio de rehabiltación durante las primeras horas del tratamiento con hemodiálisis en la Unidad renal (26).

Relación entre ERC y limitación funcional Se debe definir el término "función: funcionar/operar en la forma apropiada o particular; habiliadad de desempeñarse de forma segura y efectiva en actividades de autocuidado que son necesarias paa la vida diaria" (27).
El deterioro muscular es una disfunción fisiológica generada por anormalidades estructurales como atrofia, debilidad o reducción de la resistencia. En pacientes con ERC, la función renal reducida produce una retención de solutos urémicos, que llevan a inflamación, estrés oxidativo y resistencia a la insulina, promoviendo una alteración del metabolismo mitocondrial a nivel musculoesquelético. Se activan entonces las vías proteolíticas promoviendo atrofia muscular. Conforme progresa la enfermedad, la disfunción muscular genera mayor limitación de movilidad y pérdida de independencia funcional. Las limitaciones funcionales son las restricciones físicas básicas (caminar, subir escaleras, etc) (28).
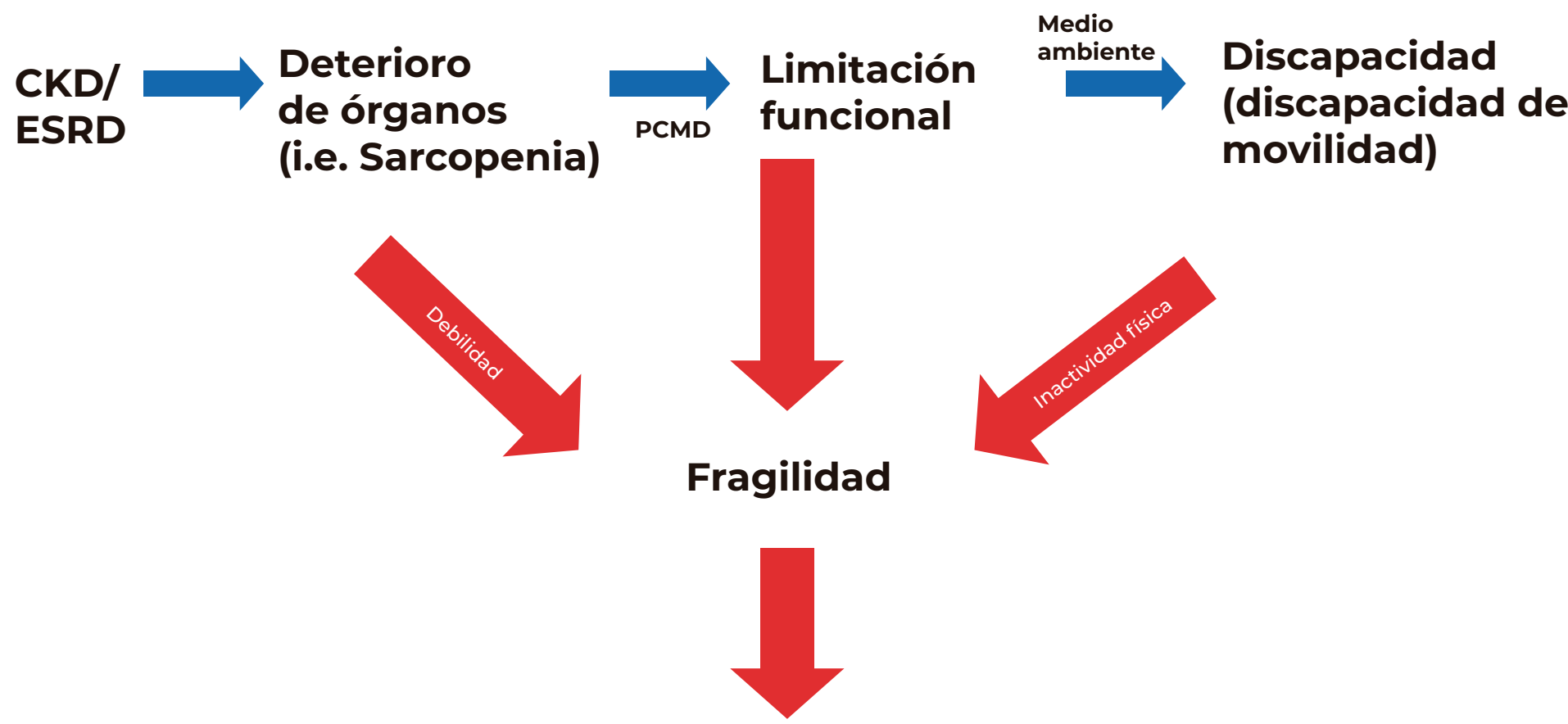

\section{Muerte o diálisis Hospitalización o muerte}

FIGURA 1 La ERC contribuye al deterioro muscular que promueve el deterioro funcional, la discapacidad de movilidad y la fragilidad. Esta figura representa la ERC en el contexto del modelo de discapacidad de Nagi. Abreviaturas: ERC, enfermedad renal crónica; ESRD, enfermedad renal en etapa terminal; PCMD, preclínico mobility disability [Color figure can be viewed at wileyonlinelibrary.com] 
Esta disfunción muscular explica el riesgo de fragilidad de los pacientes con ERC. La fragilidad se define como un síndrome clínico de vulnerabilidad caracterizado por pobre agarre (alteración muscular), baja velocidad de marcha, pobre actividad física, baja energía y pérdida de peso. La presencia de 3 o más de los anteriores indica que el paciente es frágil. El fenotipo frágil está asociado a discapacidad, hospitalización y mortalidad en todas las poblaciones (28).

El síndrome de fragilidad corresponde a un grupo de condiciones frecuentes en el adulto mayor, es decir, los síndromes geriátricos son altamente en los pacientes adultos mayores con ERC-en diálisis. Más del 50\% dependen de otros para AVD y más del $95 \%$ reciben ayuda para AVD. lo cual se relaciona con resultados adversos como hospitalizaciones, institucionalización y muerte (27).

Estudios sugieren que los pacientes con ERC tienen más probabilidades de ser frágiles y un rápido declive en la función física (3).

Es entonces esencial realizar un tamizaje temprano para encontrar aquellos pacientes con mayor riesgo de discapacidad por inmovilidad. Las limitaciones en la movilidad pueden valorarse con auto reportes o pueden verse objetivamente mediante pruebas de desempeño como la velocidad de marcha, Time up and go y la caminata de 6 minutos. En conjunto, permiten determinar de forma temprana cuáles pacientes están en riesgo de fragilidad y se beneficiarían de una valoración médica inmediata y de una referencia a terapia física para mejorar la fuerza muscular y el balance, previo al inicio de ejercicio (28).

Además influye en la fatiga que reporta la población con ERC, alrededor del $90 \%$, lo que definitivamente influye en el compromiso en sus actividades de vida diaria regulares $y$ dificultades para sus actividades rutinarias. Es decir, impacta en la participación y disfrute de cada día de vida.

\section{Autorreportes de la función física}

Subjetivamente, se pueden hacer 2 preguntas que nos permiten saber si el paciente percibe alguna limitación en la movilidad en su cotidianidad:

- ¿Tiene dificultad en caminhar $1 / 4$ de milla 0 subir 10 gradas?

- ¿Cambió la forma en que camina 3 cuadras?

Las preguntas que tratan acerca de modificaciones del paciente en tareas de movilidad, como caminar distancias cortas y subir gradas, son útiles para ver una alteración de la movilidad preclínica, es decir, que son limitaciones que existen solo en ambientes retadores y que aumentan el riesgo de discapacidad por inmovilidad.

También se han validado varios cuestionarios de calidad de vida para tamizar y dar seguimiento a esta población. El cuestionario de Salud SF-36 ha sido validado al español y se puede utilizar para evaluar la calidad de vida relacionada con muchas patologías y tratamientos. Sus propiedades psicométricas han sido ampliamente evaluadas para la población con IRC. Este cuestionario está compuesto por 36 ítems que valoran función física, rol físico, dolor corporal, salud general, vitalidad, función social, rol emocional y salud mental (29). Existe uma forma abreviada, la SF-12, que también ha demostrado confiabilidade en esta población. Sin embargo, existe otra escala, The Kidney Disease Quality of Life KDQOL-36-SF, que tiene dimensiones específicas para evaluar al paciente con enfermedad renal, con reproducibilidad, validez y sensibilidad confiables, y de fácil aplicación, poca complejidad y bajo costo. Esta escala está validada en varios países de habla hispana, 
por lo que se recomienda su uso para evaluar la calidad de vida en población con enfermedad renal. Los expertos recomiendan el uso de ambas escalas para valorar a los pacientes prediálisis y durante la diálisis(20).

Estas escalas contienen subescalas que permiten valorar por un lado el componente físico a través de la PCS, la cual resume el funcionamiento físico, el dolor y la salud general, y por otro lado la función física a través de la PF-10, la cual mide la función de la movilidad. Estas subescalas hacen que la SF-36 y la KDQOL tengan un valor predictivo importante para fragilidad (28).

Valoración objetiva de la limitación funcional y de la movilidad (28).

- Velocidade de marcha

$0<0.8 \mathrm{~m} / \mathrm{s}$ se asocia a muerte en ERC

o $>1 \mathrm{~m} / \mathrm{s}$ mejora sobrevivencia

- Levantarse y caminar $4 \mathrm{~m}>12$ seg se asocia a muerte en ERC

- Bateria corta de desempeño físico

o Puntaje $<8$ puntos se associa com mayor riesgo de discapacidad
Es importante notar que caminar implica a los sistemas cardiopulmonar, circulatorio, nervioso y musculoesquelético, por lo que la valoración de la marcha y su velocidad es un indicador confiable del estado de salud.

El siguiente algoritmo demuestra el tamizaje en dos pasos de pacientes con ERC que tienen alto riesgo de limitaciones funcionales. Tomado $\mathrm{y}$ modificado de (28).

Una vez que el paciente inicia la terapia física previa al ejercicio, se le deben enseñar ejercicios de:

- Fuerza

- Movilidad

- Equilibrio

- Modificar el ambiente para evitar caídas

Es importante notar que el componente de fragilidad más fácil de modificar es la inactividad física. Las intervenciones que buscan incrementar la actividad física funcionan para prevenir o para reducir la fragilidad en pacientes con ERC.
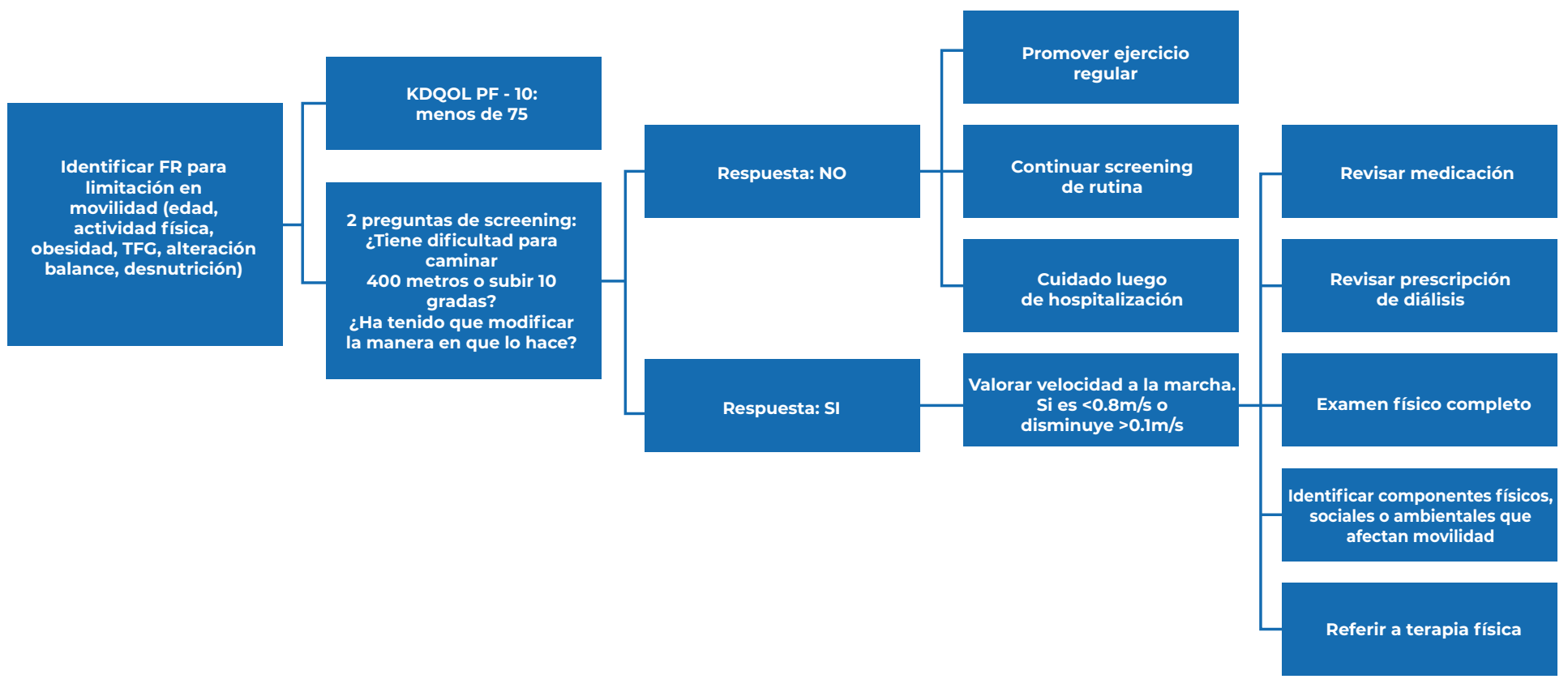
Una vía mayor por la que la ERC contribuye a la alteración del funcionamiento físico es que aumenta el nivel de fatiga, que es también la causa más frecuente de limitación para la actividad física (30).

La alteración cognitiva es más común entre los pacientes de todas las edades con enfermedad renal, que en la población general. También la alteración visual es común (27).

Las alteraciones cognitivas incluyen la concentración, planeación, memoria, tareas de conexión, no verbales, secuenciación, ajuste de desplazamiento (31).

Por lo tanto, la combinación de barreras visuales, cognitivas $\mathrm{y}$ físicas hace difícil completar las tareas diarias (27).

Además de la disminución de la fuerza muscular y las alteraciones cognitivas, la fuerza de los agarres corresponde también a cambios metabólicos que se asocian con alteración de la función física (3).

La enfermedad vascular conlleva a ERC, y la ERC acelera en gran medida la progresión de la enfermedad vascular. A la enfermedad vascular se le atribuye un tercio o la mitad de la disminución de la actividad fisica con la edad (3).

Otros factores que contribuyen en la disminución de la independencia funcional además de la debilidad muscular y dificultad para caminar, son la alteración del equilibrio, rigidez articular y neuropatía (32).

La alteración de la función física, es un marcador de riesgo para mortalidad en pacientes jóvenes con ERC (3).

En adultos mayores la disminución de la función renal y la alteración de la función física son marcadores de riesgo independientes para mortalidad (3).

\section{Intervención en rehabilitación en pacientes con ERC}

Mantener el funcionamiento físico es básico para optimizar la calidad de vida, que es el objetivo prinicpal de la rehabilitación, y por lo tanto, la intervención de un equipo de rehabilitación es fundamental de incluir en el estándar de cuidado (32).

Evaluar el funcionamiento de las personas con ERC permite identificar sus necesidades y dirigir la rehabilitación y estrategias (22).

Es importante tener como premisa que una ERC avanzada no impide ni excluye o descarta o es una barrera para una rehabilitación exitosa (3).

Basados en la evidencia que existe sobre la actividad física en pacientes con ERC, los servicios de rehabilitación renal deben enfocarse en (5):

1. Mejorar la capacidad fisiológica de reserva.

2. Aumentar la fuerza muscular y reducir la limitaciones físicas funcionales (o postergar el deterioro hasta donde sea posible).

3. Reducir el número y la severidad de síntomas específicos de ERC.

4. A largo plazo, la meta principal es incrementar y adecuar la actividad física en estos pacientes, mejorar su calidad de vida y reducir la morbimortalidad.

Es importante notar que aunque los pacientes con ERC son pacientes de alto riesgo cardiovascular, según un metaanálisis de Cochrane, no se reportan eventos adversos fatales o serios relacionados con el ejercicio. Los eventos adversos esperados son menores: hipotensión post ejercicio, fatiga $\mathrm{y}$ dolor muscular (7). 
A los adultos con ERC que aún sean independientes en AVD básicas e instrumentales, se les debe recomendar un estilo de vida activo con ejercicio físico regular, para prevenir el deterioro funcional.

Cuando ya hay disminución de habilidades y limitaciones más complejas, es más importante el abordaje multidisciplinario (1).

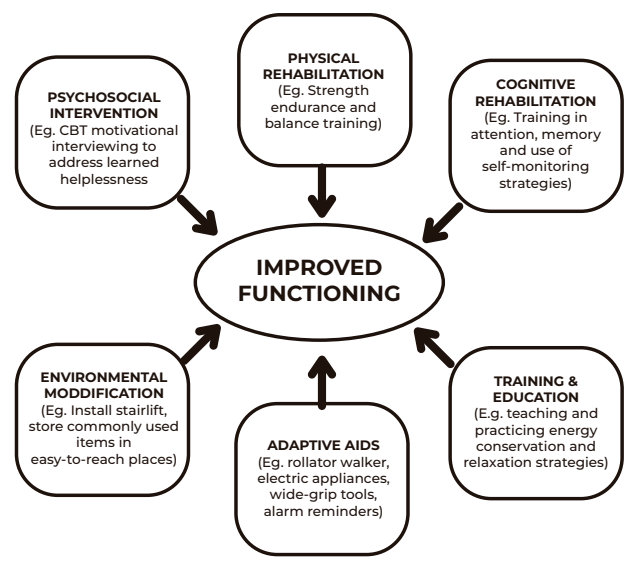

Fig. 2. Rehabilitation approaches to improving function

TABLE 1 Summary of studies examining nonpharmacologic interventions to preserve cognitive function in patients with end-stage kidney disease undergoing dialysis

\begin{tabular}{|c|c|c|c|c|}
\hline Reference & Population characteristics & Intervention description & $\begin{array}{l}\text { Measures } \\
\text { of cognition }\end{array}$ & Findings \\
\hline Baggetta et al117 & $\begin{array}{l}\text { Older HD \& PD patients aged } \\
65+\text { years from the Exercise } \\
\text { Introduction to Enhance } \\
\text { Performance in Dialysis } \\
\text { (EXCITE) trial ( } n=115, \text { mean } \\
\text { age = NA) }\end{array}$ & $\begin{array}{l}\text { Home-based, personalized } \\
\text { exercise program: } 6 \text {-min } \\
\text { walking distance and 5-time } \\
\text { sit-to-stand testing } \\
\text { (Duration of exposure: } 6 \mathrm{mo} \text { ) }\end{array}$ & KDQOL-SF & $\begin{array}{l}\text { Self-reported cognitive } \\
\text { function declined in the } \\
\text { control arm }(P=0.04) \text { and } \\
\text { remained stable in the active } \\
\text { arm }(P=0.78)\end{array}$ \\
\hline Manfredini et al114 & $\begin{array}{l}\text { Adult dialysis patients in the } \\
\text { Exercise Introduction to } \\
\text { Enhance Performance in } \\
\text { Dialysis (EXCITE) trial }(n=296 \text {, } \\
\text { mean age }=\text { NA) }\end{array}$ & $\begin{array}{l}\text { Home-based, personalized } \\
\text { exercise program: } 6 \text {-min } \\
\text { walking distance and 5-time } \\
\text { sit-to-stand testing } \\
\text { (Duration of exposure: } 6 \mathrm{mo} \text { ) }\end{array}$ & KDQOL-SF & $\begin{array}{l}\text { Walking exercise } \\
\text { (intervention) versus normal } \\
\text { activity (controls) experienced } \\
\text { improvements in } \\
\text { self-reported cognitive } \\
\text { function }(P=0.04) \text { and quality } \\
\text { of social interaction }(P=0,01)\end{array}$ \\
\hline $\begin{array}{l}\text { McAdams- } \\
\text { DeMarcoet al115 }\end{array}$ & $\begin{array}{l}\text { Adult HD patients of all ages } \\
\text { participating in a pilot } \\
\text { randomized controlled trial ( } n \\
=20, \text { mean age }=50.8 \text { ) }\end{array}$ & $\begin{array}{l}\text { intradialytic CT } \\
\text { (Tablet-based brain } \\
\text { games), ET (foot peddlers), } \\
\text { or standard of care (SC) } \\
\text { (Duration of exposure: } 3 \\
\text { mo) }\end{array}$ & $\begin{array}{l}\text { 3MS } \\
\text { TMTA } \\
\text { TMTB }\end{array}$ & $\begin{array}{l}\text { Patients with SC experienced } \\
\text { psychomotor speed and } \\
\text { executive function decline by } \\
3 \text { mo; this decline was not } \\
\text { seen in both intervention } \\
\text { arms }(C T \text { or } E T \text {, all } P>0,05 \text { ) }\end{array}$ \\
\hline Stringuetta et al72 & $\begin{array}{l}\text { Adult HD patient of all ages } \\
\text { participating in pilot } \\
\text { randomized controlled trial ( } \mathrm{n} \\
=30, \text { mena age }=\mathrm{NA} \text { ) }\end{array}$ & $\begin{array}{l}\text { Intradialytic ET (cycling) } \\
\text { (Duration of exposure: } 4 \\
\text { mo) }\end{array}$ & $\begin{array}{l}\text { MMSE } \\
\text { Transcranial } \\
\text { Dopplet }\end{array}$ & $\begin{array}{l}\text { Patients with ET improved in } \\
\text { cognitive function ( } P=0,001) \\
\text { and basiliar maximum blood } \\
\text { flow velocity ( } P=0.029 \text { ) } \\
\text { compared to control group. } \\
\text { ET group also had greater } \\
\text { proportion of arteries with } \\
\text { increased flow velocity ( } P= \\
0,038 \text { ) }\end{array}$ \\
\hline
\end{tabular}

Abbreviations: 3MS, Modified Mini-Mental State Exam for global cognitive function; CT, cognitive training: ET, exercise training: HD, hemodialysis; XDQOL-SF, Kidney Disease Quality of Life Short Form questionnaire with cognitive function subcomponents; MMSE, Mini-Mental State Exam; NA not available, SC, standard of case; TMTA and TMTB, Trails, Making Tests Parts A and B for processing speed; TMTB - TMTA, Trail Making Test B minus Trails Making Test A for executive function. 


\section{Individual Level}

Risk Factors

Cognitive Abilities

Motor Abilities

Cardiovascular Disease

Diabetes

History of Stroke

Medications

Nutritions

Demographics

Genetics

\section{Interventions}

Home-based Exercise

Training

Intradialysis Exercise

Training

Intradialysis Cognitive

Training

Combined Cognitive \&

Excercise Training?
Short term Outcomes

+ Cognitive Function (Global \&

Domain-specific)

+ Cerebral Blood Flow

t Brain Volume

† Motor Function

\section{Long-term}

Outcomes

† Medication Adherence † Health Related Quality of Life

† Graft-Loss

† Dementia

$\uparrow$ Mortality

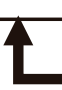

Componentes clave en el plan de rehabilitación basado en ejercicio

\section{Valoración}

Se debe realizar una historia detallada de las condiciones crónicas, de los factores de riesgo y del tratamiento actual. Se debe valorar salud física mental y social, con medidas objetivas para monitorizar progresión y reajustar la prescripción. Se debe enfatizar en los problemas en salud que pueden mejorar con el ejercicio (5).

Los test de calidad de vida citados previamente permiten tamizar el estado funcional del paciente previo a la terapia y luego darle seguimiento con las intervenciones de rehabilitación.

También se recomiendan instrumentos de valoración cognitiva global en pacientes con ER terminal al menos 1 vez cada 6 meses. El test Minimental modificado se puede utilizar de base, pero el MoCA (Montreal Cognitive Assessment) se reconoce como una herramienta más sensible para detectar aquellos pacientes con déficit cognitivo leve (20).

Consejo de actividad física y prescripción de ejercicio (5) (8)

Todas las personas con ERC deben ser consideradas como candidatos para intervenciones en prescripción de ejercicio.
Todos los ERC 4-5 deben tener al menos una sesión con un profesional en rehabilitación cada 6 meses o más, según la necesidad, para valorar la función física, el estado de actividad física y para cambiar algún elemento en esta de ser necesario. Se debe dar información escrita sobre oportunidades locales para hacer actividad física o referir a un centro de rehabilitación en la comunidad que sea estructurado y supervisado. Los planes de ejercicio verbales deben escribirse en expediente con metas personales claras.

Se debe priorizar la prescripción de ejercicio estructurado y supervisado en pacientes con alguna de las siguientes características:

- Pobre funcionamiento en AVD

- Debilidad severa

- Síntomas de disconfort respiratorio y cardiovascular en AVD

- Miedo del ejercicio y falta de confianza

- Ausencia de experiencia previa en ejercicio

- Alteraciones psicológicas serias

Los pacientes con ERC que tienen sus comorbilidades bien controladas y que no cumplen con los criterios anteriores, deben recibir una valoración inicial para entregarles una referencia para un programa de actividad física en la comunidad y un plan de ejercicios y actividad física escrito hasta la próxima cita. 


\section{Seguridad y contraindicaciones}

El ejercicio en esta población es seguro (8).

Es necesario considerar ciertas precauciones de seguridad (5) (33):

- Posponer el ejercicio si la glicemia $>250 \mathrm{mg} / \mathrm{dl}$ o <100mg/dl

- Pacientes que tienden a hipoglicemia deben hacerse glicemia x mm previo, durante y después del ejercicio, y deben tener snacks de alto índice glicémico a mano

- Posponer o detener el ejercicio si los pacientes tienen síntomas de TVP como edema, enrojecimiento o dolor inusual en la pierna.

- Si hay heridas abiertas se debe evitar natación y actividades de descarga de peso si hay ampollas

- Se les debe enseñar cómo evitar maniobras de valsalva, particularmente durante programa de fortalecimiento

- Posponer o detener el ejercicio en caso de mareo, cefalea severa, FC fluctuante, respuestas en la presión arterial

- Consultar con un médico nefrólogo sobre interacciones medicamentosas en caso de que los pacientes presenten regularmente hipotensiones y sx luego de diálisis y ejercicio

- La valoración de función física en pacientes en hemodiálisis debería hacerse en días sin diálisis

- El brazo con la fístula arteriovenosa puede ejercitarse siempre y cuando la herida esté sana y el paciente no esté conectado a una máquina de diálisis

- Para los pacientes en diálisis peritoneal puede ser más cómodo ejercitarse cuando el peritoneo está vacío y así evitar un aumento de presión en el diafragma que puede ser incomodo o causar fugas en el sitio del catéter.
Por su parte, existen contraindicaciones absolutas para realizar ejercicio en ERC (33):

- EAC inestable

- ICC descompensada

- Arritmias no controladas

- HTPulmonar severa

- Estenosis aortica severa y sintomática

- HTA no controlada >180/110

- Disección de aorta

\section{Prescripción del ejercicio}

Se debe enfatizar en dar consejo sobre cómo aprovechar las oportunidades de actividad física diaria. La prescripción de ejercicio debe basarse en la valoración de la función física y en la motivación del paciente. La evidencia sugiere un mínimo de volumen de ejercicio/actividad física de $1000 \mathrm{kcal}$ por semana para alcanzar beneficios en salud (7) (34).

La prescripción del ejercicio se basa en el principio FITT (Frecuencia, Intensidad, Tiempo y Tipo):

- Frecuencia: pacientes estables en hemodiálisis pueden seguir con seguridad un plan estructurado durante las primeras 2 horas de diálisis. Se recomienda ejercicio supervisado 3 veces por semana durante 4-6 meses para todos los estadíos. Se aconseja la participación en actividad física diaria, la mayoría de días de la semana (7).

- Intensidad: los ejercicios aeróbico o de resistencia moderados durante la diálisis (sobre todo los ejercicios aeróbicos) o en días de no diálisis en todos los estadíos de ERC es seguro y mejora la capacidad de reserva cardiovascular y la fuerza muscular (5).

- Tiempo: el nivel inicial del paciente y su estado clínico determinarán el tiempo de ejercicio que es seguro. Se recomienda de 30-90 minutos por sesión (7).

- Tipo: no existen restricciones en cuanto al tipo de ejercicio que se puede practicar con ERC. Se enfatiza en las recomendaciones de seguridad(4). Se recomiendan ejercicios que utilicen grandes grupos musculares de forma continua, como bicicleta, caminar o trotar (7) (35) (8). 


\section{Cuadro 1}

\begin{tabular}{|c|c|c|c|}
\hline Tipo & Frecuencia & Intensidad & Tiempo \\
\hline Aeróbico & $\begin{array}{l}\text { Semana 1-2: } \\
\text { 2/semana } \\
\text { Semana 3-5: } \\
\text { 3x/semana }\end{array}$ & $\begin{array}{l}\text { Semanas 1-2: moderado } \\
\text { (Borg 11-13), 55-70\% FCMax } \\
\text { Semanas: 3-5: moderado } \\
\text { (Borg 11-16), 55-90\% FCMax } \\
60-70 \& \text { de } 1 \text { RM o } 5 \text { RM }\end{array}$ & $\begin{array}{l}20 \mathrm{~min} / \mathrm{d} \\
\text { Ciclos intermitentes } 3-5 \mathrm{~min}\end{array}$ \\
\hline Resistencia & 2 veces por semana & $60-70 \%$ de 1 RM o 5 RM & $\begin{array}{l}\text { Min } 1 \text { set } 10-15 \text { reps, aumento gradual } \\
2-4 \text { sets } \\
8-10 \text { ejercicios diferentes para grandes } \\
\text { grupos musculares, descanso } 2-3 \text { min } \\
\text { entre sets, }>48 \text { h entre sesiones }\end{array}$ \\
\hline
\end{tabular}

Flexibilidad 5 veces por semana

$10 \mathrm{~min} / \mathrm{d}$

\section{Cuadro 2}

\begin{tabular}{lllll} 
Nivel basal & Frecuencia & Intensidad & Tiempo & Tipo \\
\hline Sedentarismo & $\begin{array}{l}\text { 3-5 por } \\
\text { semana }\end{array}$ & $\begin{array}{l}\text { Leve } \\
\text { EEP 3-6/10 }\end{array}$ & 20/30 min/d & $\begin{array}{l}\text { Caminata 3000- } \\
\text { 3500 pasos }\end{array}$ \\
\hline $\begin{array}{l}\text { Actividad física } \\
\text { mínima-no ejercicio- } \\
\text { desacondicionamiento } \\
\text { moderado-alto }\end{array}$ & $\begin{array}{l}3-5 \text { por } \\
\text { semana }\end{array}$ & $\begin{array}{l}\text { Leve-moderado } \\
\text { EEP 3-6/10 }\end{array}$ & $30 / 60 \mathrm{~min} / \mathrm{d}$ & $\begin{array}{l}\text { Caminata 3000- } \\
\text { 4000 pasos }\end{array}$ \\
\hline $\begin{array}{l}\text { Actividad física } \\
\text { esporádica- ejercicio }\end{array}$ & $\begin{array}{l}\text { 3-5 por } \\
\text { subóptimo - } \\
\text { desacondicionamiento } \\
\text { leve-moderado }\end{array}$ & Moderado-intenso & $30 / 90$ min/d & $\begin{array}{l}\text { Caminata }>4000 \\
\text { pasos, meta 5,400- } \\
7900 \text { y 150min/semana } \\
\text { intensidad moderada }\end{array}$ \\
\hline
\end{tabular}

El cuadro 1 resume las recomendaciones de ejercicio para pacientes con ERC y ER terminal. Adaptado de (33).

El cuadro 2 resume las recomendaciones de ejercicio aeróbico basándose en el nivel de actividad de base. Adaptado de (33).

Se debe alentar la progresión y monitorizar la adherencia y los retrocesos. Para ello se recomienda revalorar la función física cada 4-6meses para ajustar el plan de tratamiento y dar retroalimentación. Es necesario entonces documentar metas, resultados y progreso (35) (34) (5).

\section{Educación y soporte al paciente} Se debe orientar al paciente en unirse a grupos comunitarios y asociaciones de personas con ERC para poder hacer un buen uso de los recursos y del material educativo. También se debe procurar información escrita y alentar a los pacientes activamente a que se involucren en todas las actividades seguras como sea posible (35) (34) (5).

Barreras para la actividad física y servicios de rehabilitación en el paciente con enfermedad renal crónica (34)

Especificaciones para poblaciones especiales AM con ERC en estadíos tempranos: 
Table 3. All emerged themes of barriers to promotion/provision of PA and rehabilitation services in units as reported by 120 respondents

Money/funding

Lack of time

Lack of qualified personnel (physio or other exercise professional) for this role

Lack of physical resources or difficulty with existing resources(e.g. dialysis beds, exercise

$32(26.6)$

equipment)

$20(16.6)$

Lack of interested /motivated patients duw to ill health and lack of awareness

$14(11.6)$

Prioritisation of other services/lack of vision

$14(11.6)$

Lack of motivated medical staff

$10(8.3)$

Lack of leadership and professional advise on how to organise a unit with rehab in mind

Lack of space

Lack of hard research evidence, lack of knowledge about available offered services within organisations, culture, obstruction by health and safety management

En pacientes con ERC temprana, con pocas comorbilidades y con limitaciones funcionales leves, se indican ejercicios aeróbicos y de resistencia de moderado-alta intensidad (1) (36).

\section{AM con ERC con múltiples comorbilidades y discapacidad leve}

Se deben planear intervenciones específicas cuando existen comorbilidades como artritis, sarcopenia, falla cardiaca y alteraciones neurológicas que contribuyen a la discapacidad.

Estos abordajes deben planearse según las preferencias de cada paciente, individualizándolo, y tomando en cuenta su habilidad funcional. El abordaje debe ser más estructurado e interdisciplinario conforme avance el declive funcional.

El programa de ejercicio debe incluir (1):

- Ejercicio aeróbico

- Ejercicio de resistencia

- Facilitación neuromuscular propioceptiva

- Ejercicios de equilibrio

- Ejercicios de flexibilidad de extremidades y de la columna

- Entrenamiento de marcha
Terapia ocupacional se encarga de la adaptación del ambiente y del hogar para los AM que están en riesgo de caídas, que es la principal causa de lesión y morbilidad asociada en AM. Las caídas se asocian a una disminución de la movilidad, a hospitalización, a síndromes depresivos, a declive funcional, a disminución en la participación y a pobre calidad de vida. Los programas de prevención incluyen valoración de peligros en casa por un equipo entrenado, retiro o modificación de peligros encontrados, instalación de aditamentos tipo barandas, agarraderas y mejorías en la iluminación. De hecho, a todos los pacientes (con o sin ERC) AM en riesgo de caídas se les debe ofrecer un programa de ejercicios que incluya entrenamiento de balance, marcha y fortalecimiento (1).
AM frágiles o pacientes en estadio terminal de la enfermedad
Los AM frágiles o terminales con ERC también se benefician de actividad física regular que incluya ejercicio aeróbico y entrenamiento de resistencia. Entre los factores de fragilidad uno de los más importantes es la pérdida de masa muscular y existe evidencia de que el ejercicio aeróbico aumenta la masa muscular de miembros inferiores en los AM (1) (36). 
El ejercicio debe prescribirse según las limitaciones y la habilidad residual del paciente, enfocándose en las prioridades del paciente y en la participación de los cuidadores. Además de ejercicio aeróbicos, de resistencia, de propiocepción y equilibrio, de flexibilidad y del entrenamiento de marcha, se debe sumar también los ejercicios de terapia ocupaciones y la rehabilitación cognitiva y de lenguaje (1) (36).

Es fundamental considerar la terapia ocupacional cuando hay afección para las AVD, además de prescribir aditamentos $\mathrm{y}$ aparatos que asistan en las AVD: palos para vestirse (dressing stick), calzador largo, esponja con agarradera larga, gancho para botones, asiento de baño, entre otros. Estos elementos han demostrado ser efectivos en minimizar el gasto energético, en aumentar la eficiencia del tiempo y en disminuir la frustración, mejorando así la función en el autocuidado y en las AVD (1).

En términos generales, en los programas de rehabilitación de pacientes adultos mayores con ERC debe considerarse la prevención caídas, entrenamiento con ejercicio-prehabilitación proactiva, rehabilitación basada en el hogar y telerehabilitación (27) (36).

\section{REHABILITACION OF THE GERIATRIC DIALYSIS PATIENT}

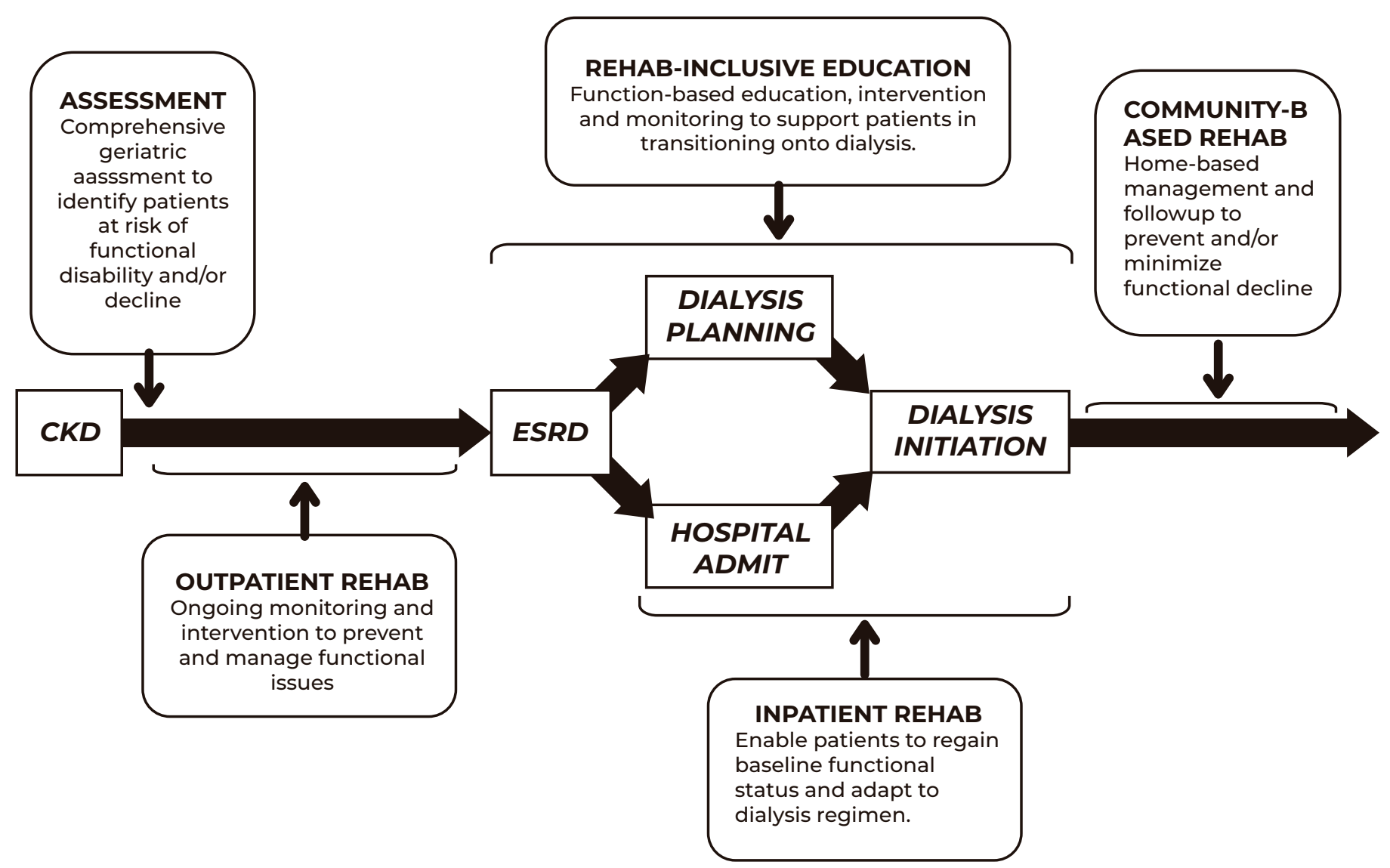

Fig. 4. Opportunities to include geriatric rehabilitation in the current model of geriatric dialysis care Fuente: (27). 
Tabla 1. Estrategias de rehabilitación en ancianos con ERC y ERT

\section{Early stage of CKD}

physical activity including aerobic and resistance exercises producing positive effects on cardio-respiratory fitness, physical function and self-reported health.

moderate to high-intensivity strength training improves physical performance, muscle mass and quality of life

individual patient-centered approach:

(1) physical activity including aerobicexercises

(2) resistance exercises

(2) proprioceptive neuromuscular facilitation

(2) posture and balance control

(-) joinsts mobilization and spine flexibility exercises

(-) endurance, muscle strength and muscular stretching gait training

individualized patient-centered approach according functional limitations and residual ability;

Frail CKD/ESRD elder dweiling-people address limitations and focus on patients priority and family or caregivers participation:

golas-oriented interventions and functional areas of interventions with supervised sessions;

patients and family educations:

() physical activity including aerobics exercises

(-) proprioceptive neuromuscular facilitation

(-) posture and balance control

(-) joints mobilization

(-) muscular stretching

(-) gait training

(-) occupational therapy speed and cognitive rehabilitation comprehensive multi-dimensional strategy and goal-oriented approach; discussion regarding goals of care and advance care planning ought to be common place for such patients; specialized ward and interdisciplinary care team; geriatric hemodialysis rehabilitation care
Frail CKD/ESRD people requiring hospitalizations

\section{Pacientes en hemodiálisis}

Los pacientes en hemodiálisis tienen una tolerancia al ejercicio, una capacidad funcional, una resistencia y una fuerza considerablemente menor que pacientes con ERC que no requieren de una terapia de reemplazo renal, así como tienen mayor pérdida muscular y fatiga. En ellos se pueden 
aplicar estrategias de rehabilitación con las mismas recomendaciones y contraindicaciones que los AM y que los pacientes sin diálisis según la American College of Sports Medicine and the American Heart Association (1).

Los pacientes en hemodiálisis tienen una caapcidad para el ejercicio menor del $40-50 \%$ de la predicha para sujetos normales (mucho menor que en otras formas de enfermedad renal crónica) (30).

Los beneficios asociados al ejercicios en estos pacientes son mejorías en el VO2 pico, en la función cardiaca, en la calidad de vida y en la actividad simpático adrenal. También se reportan mejorías en la masa muscular, en el área muscular de los cuádriceps, en la extensión de rodilla, en la fuerza de abducción y en la flexión de cadera.

El entrenamiento se puede dar en tiempo de no diálisis, en casa o en consulta externa, así como durante la diálisis (entrenamiento intradialítico)(1). Mejora funcionamiento físico, fitness cardiorespiratorio, riesgo cardiovascular. Los pacientes físicamente activos a la hora de iniciar diálisis sobreviven más que los sedentarios (4).

Los ejercicios de resistencia pueden ser con máquinas sofisticadas (press de piernas o pesas) o con elementos simples como bandas elásticas para utilizar durante diálisis.

Todavía existen dudas acerca del programa más adecuado para obtener beneficio, pero no se han encontrado diferencias entre programas de ejercicio intradiálisis vs programas para el hogar (1).

Se ha visto que los pacientes más frágiles y con mayor discapacidad son los que más necesitan rehabilitación física como parte de su cuidado. El inicio de diálisis se asocia con deterioro funcional independientemente de la edad, del género $\mathrm{y}$ del estado funcional previo, y este fenómeno es más dramático en el AM.

En efecto, los AM en diálisis tienen $50 \%$ menos capacidad funcional que los de su misma edad y género, sin ERC. Solo $13 \%$ de estos pacientes luego de 1 año de hemodiálisis mantienen $\mathrm{su}$ función física estable.

El deterioro funcional que ocurre en AM en hemodiálisis produce alteraciones de la movilidad, caídas, fracturas y limitaciones funcionales que predisponen a estos pacientes a mayor necesidad de cuidados médicos e institucionalización a largo plazo.

Cabe resaltar que la mayor parte de los pacientes AM en hemodiálisis tienen además comorbilidades que producen limitación funcional severa y deterioro de calidad de vida por si solas: DM, EAC, ICC, AR, dolor, PNP, alteraciones afectivas y cognitivas (1).

Es por todo esto que la intervención multidimensional orientada en metas es lo que se recomienda actualmente para esta población. De esta manera se han propuesto unidades especializadas de rehabilitación durante la diálisis, que permita un cuidado multidisciplinario con expertos en rehabilitación, geriatría y nefrología para que el paciente y sus familiares-cuidadores tengan metas de rehabilitación realistas: alcanzar nivel de independencia acorde con el estado previo del paciente, y aclarar que no se pueden revertir algunas discapacidades permanentes (1). 
El siguiente cuadro resume la progresión de la prescripción de ejercicio para pacientes adultos mayores en hemodiálisis (5).

Table L Indicative initial 3 month exercise prescription plan for an elderly patient on haemodialysis.

\begin{tabular}{|c|c|c|c|c|}
\hline Primary and secondary outcomes & Goals & $\begin{array}{l}\text { Exercise treatment plan } \\
\text { (monitor tolerance and vital signs) }\end{array}$ & Progression & $\begin{array}{l}\text { Additional } \\
\text { intervention } \\
\text { components } \\
\end{array}$ \\
\hline 1. Severe muscle weakness & $\begin{array}{l}\text { Improve lower body } \\
\text { strength }\end{array}$ & \multirow{2}{*}{$\begin{array}{l}\text { F: } 2 / \text { week } \\
\text { I: } 60 \% \text { of peak workload achieved at } \\
\text { assessment (if testing progressed } \\
\text { without complications) } \\
\text { T: Cycling } \\
\text { T: } 15 \text { min in total in } 5+5+5\end{array}$} & \multirow[t]{2}{*}{ Week 1-2 } & \\
\hline $\begin{array}{l}\text { 2.Severe difficulty to perform } \\
\text { basic ADLs }\end{array}$ & $\begin{array}{l}\text { Increase exercise } \\
\text { tolerance }\end{array}$ & & & \\
\hline \multirow[t]{7}{*}{ 3. Low threshold exertional fatigue } & & $\begin{array}{l}\text { F: } 3 / \text { week } \\
\text { I: } 60 \% \text { of peak workload } \\
\text { T: cycling } \\
\text { T: } 20 \text { min ( } 10+10 \text { or any other } \\
\text { combination) }\end{array}$ & Weeks 3-5 & \\
\hline & & $\begin{array}{l}\text { F: } 3 / \text { week } \\
\text { I: } 60 \% \text { of peak workload } \\
\text { T: cycling } \\
\text { T: } 20 \text { min }\end{array}$ & \multirow[t]{2}{*}{ Weeks 6-9 } & \multirow[t]{2}{*}{$\begin{array}{l}10-20 \text { sit-to-stand } \\
\text { transfets at home } \\
4 \text { days/week }\end{array}$} \\
\hline & & $\begin{array}{l}\text { F: } 2 / \text { week } \\
\text { I: } 60 \% 5 R M . \\
\text { T: resistance weights } \\
\text { T: } 10 \text { min with tests ( } 2 \text { sets } x \\
8 \text {-reps dynamic leg exercises) }\end{array}$ & & \\
\hline & & $\begin{array}{l}\text { F: } 3 / \text { week } \\
\text { I: } 70 \% \text { of peak workload } \\
\text { T:cycling } \\
\text { T: } 30 \text { mit) }\end{array}$ & \multirow[t]{4}{*}{ Weeks 10-13 } & \multirow[t]{4}{*}{$\begin{array}{l}2 \times 20 \text { sit-to-stand } \\
\text { transfers } 4 \text { days! } \\
\text { week unsupported }\end{array}$} \\
\hline & & F: 2/week & & \\
\hline & & I: 70\% 5RM & & \\
\hline & & $\begin{array}{l}\text { T: resistance weights } \\
\text { T: } 15 \text { min with tests ( } 3 \text { sets x 8-10 } \\
\text { reps (dynamic leg exercise) }\end{array}$ & & \\
\hline Re-assess & Interpret & Monitor/Adjust/C,ontinue & Feedback & Advise \\
\hline
\end{tabular}

Note: ADL Activities of daily living, FITT: Frequency, Intensity, Type, Time, RM: Repetition maximum

Table 1. Potential Impairments and Functional Limitations and Related PT and OT Treatment Interventions

Impairment/Functional Limitations Possible Treatment Interventions

Loss of balance

Muscle weakness

Joint ROM limitations

Generalized pain and parasthesias

Low back pain

Difficulty ambulating

ADL limitations
Therapeutic activities: proprioceptive, kinesthetic, stimulation of joint receptors with joint accessory motion

Therapeutic exercise, neuromuscular re-education, resistance exercise, PNF

Passive/active ROM, joint mobilization, soft-tissue techniques, and PNF (reciprocal inhibition for hypertonic opposing muscles, ie, triceps activation to "turn off" biceps with elbow flexion hypertonicity or contracture)

Soft-tissue techniques to decrease spasms and provide sensory input (vibration, different textures, varying pressures), ultrasound to improve blood flow and tissue temperature, electrical modalities to improve blood flow, contract and relax musculature, and joint mobilizations to improve joint nutrition and awareness

Same as aboye, but including positioning, postural awareness, and deep abdominal strengthening.

Dynamic balance, joint ROM of motions used in ambulation, and upperextremity strengthening for patients who use an assistive device Fine-motor control and strengthening, therapeutic activities mimicking components of ADLs, and PNF to facilitate joint control and motion in functional positions and movements

Abbreviation: PNF, proprioceptive neuromuscular facilitation

Fuente: (32) 
El entrenamiento cognitive debe de ser una parte integral para el manejo de las secuelas cognitivas de la ERC, en conjunto con el ejercicio prescrito.

\section{Prehabilitación y trasplante renal}

La función física reducida previa al trasplante se asocial de forma significativa a un riesgo elevado de hospitalización post-trasplante y de mortalidad. En efecto, existe una correlación entre el estado functional preoperatorio y los resultados perioperatorios. Existe poca literature respect al efecto de la prehabilitación en cirugías de trasplante renal, mientras que la gran parte de los estudios de prehabilitación por cirugías mayores se han realizado en cirugías ortopédicas y cardiotorácicas. En general, los estudios existentes reportan una majoría modesta (menor al 15\%) en la condición cardiopulmonary luego de un programa de ejercicio de 3-8 semanas previo al trasplante renal, comparado con los grupos controles. A pesar de esto, sabiendo que el decline functional y el desgaste muscular es importante en pacientes con ERC, aquellos pacientes en espera de un traspalnte de ri\{ón deberia estar inscrito en un programa de prehabilitación que evite estos efecto indeseables. Faltan estudios que comprueben los beneficios de estos programas (37).

\section{Conclusiones}

La ERC es un problema de salud pública asociada a deterioro funcional y discapacidad en quien la padece. A finales del 2017 la CCSS inició la implementación del Protocolo para la atención de la persona con enfermedad renal crónica en la red de servicios institucional, dirigido a la prevención, al diagnóstico temprano y tamizaje. Sin embargo, este protocolo no incluye la rehabilitación de los pacientes com ERC, independentemente de su estadío o manejo. La evidencia ha demostrado que las intervenciones en ejercicio y la actividad física son efectivas en disminuir la tasa de progresión de ERC, la mortalidad y las hospitalizaciones; además mejoran la sensación de bienestar, confianza, calidad de vida y desempeño físico de los pacientes con ERC, independientemente del estadío. Es por esto que los servicios renales deben incorporar opciones de rehabilitación con programas integrales de ejercicio físico y cognitivo, en los que el médico rehabilitador coordina a un equipo interdisciplinario. Faltan estudios aún que justifiquen los programas de prehabilitación en pacientes con trasplante renal, así como otros que demuestren la mejor dosis de ejercicio y cuál tipo es el más adecuado en estos pacientes (aeróbico, de resistencia, combinados).

\section{Bibliografía}

1. Intiso D. The rehabilitation role in chronic kidney and end stage renal disease. Kidney Blood Press Res. 2014 Jul 29;39 (2-3):180-8.

2. Smyth A, Glynn LG, Murphy AW, Mulqueen J, Canavan M, Reddan DN, et al. Mild chronic kidney disease and functional impairment in community-dwelling older adults. Age Ageing. 2013 Jul;42(4):488-94.

3. Doyle EM, Sloan JM, Goodbrand JA, McMurdo MET, Donnan PT, McGilchrist MM, et al. Association between kidney function, rehabilitation outcome, and survival in older patients discharged from inpatient rehabilitation. Am J Kidney Dis. 2015 Nov;66(5):768-74.

4. Rossi AP, Burris DD, Lucas FL, Crocker GA, Wasserman JC. Effects of a renal rehabilitation exercise program in patients with CKD: a randomized, controlled trial. Clin J Am Soc Nephrol. 2014 Dec 5;9(12):2052-8. 
5. Koufaki P, Greenwood S, Painter P, Mercer T. The BASES expert statement on exercise therapy for people with chronic kidney disease. J Sports Sci. 2015 Mar 25;33(18):1902-7.

6. O'Hare AM, Tawney K, Bacchetti P, Johansen KL. Decreased survival among sedentary patients undergoing dialysis: results from the dialysis morbidity and mortality study wave 2 . Am J Kidney Dis. 2003 Feb;41(2):447-54.

7. Heiwe S, Jacobson SH. Exercise training for adults with chronic kidney disease. Cochrane Database Syst Rev. 2011 Oct 5;(10):CD003236.

8. Gould DW, Graham-Brown MPM, Watson EL, Viana JL, Smith AC. Physiological benefits of exercise in pre-dialysis chronic kidney disease. Nephrology (Carlton). 2014 Sep;19(9):519-27.

9. Takaya Y, Kumasaka R, Arakawa T, Ohara T, Nakanishi M, Noguchi T, et al. Impact of cardiac rehabilitation on renal function in patients with and without chronic kidney disease after acute myocardial infarction. Circ J. 2014;78(2):377-84.

10. Hama T, Oikawa K, Ushijima A, Morita N, Matsukage T, Ikari Y, et al. Effect of cardiac rehabilitation on the renal function in chronic kidney disease - Analysis using serum cystatin-C based glomerular filtration rate. IJC Heart \& Vasculature. 2018 Jun;19:27-33.

11. Pei G, Tang Y, Tan L, Tan J, Ge L, Qin W. Aerobic exercise in adults with chronic kidney disease (CKD): a meta-analysis. Int Urol Nephrol. 2019 Oct;51(10):1787-95.

12. Sheng K, Zhang P, Chen L, Cheng J, Wu C, Chen J. Intradialytic exercise in hemodialysis patients: a systematic review and meta-analysis. Am J Nephrol. 2014 Dec 9;40(5):478-90.
13. Müller-Ortiz H, Pedreros-Rosales C, Vera-Calzaretta A, González-Burboa A, Zúñiga-San Martín C, Oliveros-Romero MS. [Exercise training in advanced chronic kidney disease]. Rev Med Chil. 2019 Nov;147(11):1443-8.

14. Greenwood SA, Castle E, Lindup H, Mayes J, Waite I, Grant D, et al. Mortality and morbidity following exercise-based renal rehabilitation in patients with chronic kidney disease: the effect of programme completion and change in exercise capacity. Nephrol Dial Transplant. 2019 Apr 1;34(4):618-25.

15. Morishita S, Tsubaki A, Shirai N. Physical function was related to mortality in patients with chronic kidney disease and dialysis. Hemodial Int. 2017 Apr 18;21(4):483-9.

16. Tentori F, Elder SJ, Thumma J, Pisoni RL, Bommer J, Fissell RB, et al. Physical exercise among participants in the Dialysis Outcomes and Practice Patterns Study (DOPPS): correlates and associated outcomes. Nephrol Dial Transplant. 2010 Sep;25(9):3050-62.

17. Koufaki P, Mercer TH, Naish PF. Effects of exercise training on aerobic and functional capacity of end-stage renal disease patients. Clin Physiol Funct Imaging. 2002 Mar;22(2):115-24.

18. Hargrove N, Tays Q, Storsley L, Komenda P, Rigatto C, Ferguson T, et al. Effect of an exercise rehabilitation program on physical function over 1 year in chronic kidney disease: an observational study. Clin Kidney J. 2020 Feb;13(1):95-104.

19. Bronas UG, Puzantian H, Hannan M. Cognitive impairment in chronic kidney disease: vascular milieu and the potential therapeutic role of exercise. Biomed Res Int. 2017 Apr 19;2017:2726369. 
20. Chu NM, McAdams-DeMarco MA. Exercise and cognitive function in patients with end-stage kidney disease. Semin Dial. 2019 Mar 22;32(4):283-90.

21. Sakkas GK, Giannaki CD, Karatzaferi C, Maridaki M, Koutedakis Y, Hadjigeorgiou GM, et al. Current trends in the management of uremic restless legs syndrome: a systematic review on aspects related to quality of life, cardiovascular mortality and survival. Sleep Med Rev. 2015 Jun;21:39-49.

22. de Araújo Filho JC, Cavalcanti FCB, Morais GS, Bezerra SD, Costa MJC, Marinho PÉ de M. Development of an International Classification of Functioning, Disability and Health core set for adults with chronic kidney disease undergoing hemodialysis: a scoping review protocol. JBI Evid Synth. 2020 May;18(5):1116-23.

23. Chauveau P, Lasseur C, Aparicio M. [Is there a place for the physical activity in the prevention of the fractures of chronic kidney disease patients?]. Nephrol Ther. 2012 Jul;8(4):216-9.

24. Afsar B. The relationship between intact parathyroid hormone levels and daily physical activity in hemodialysis patients. Int J Artif Organs. 2014 May;37(5):350-7.

25. Moinuddin I, Leehey DJ. A comparison of aerobic exercise and resistance training in patients with and without chronic kidney disease. Adv Chronic Kidney Dis. 2008 Jan;15(1):83-96.

26. Kouidi EJ. Central and peripheral adaptations to physical training in patients with end-stage renal disease. Sports Med. 2001;31(9):651-65.

27. Farragher J, Jassal SV. Rehabilitation of the geriatric dialysis patient. Semin Dial. 2012 Dec;25(6):649-56.
28. Roshanravan B, Patel KV. Assessment of physical functioning in the clinical care of the patient with advanced kidney disease. Semin Dial. 2019 May 1;32(4):351-60.

29. Vilagut G, Ferrer M, Rajmil L, Rebollo P, Permanyer-Miralda G, Quintana JM, et al. El Cuestionario de Salud SF-36 español: una década de experiencia y nuevos desarrollos. Gaceta Sanitaria. 2005 Apr;19(2):135-50.

30. Kutner NG, Cardenas DD, Bower JD. Rehabilitation, aging and chronic renal disease. Am J Phys Med Rehabil. 1992 Apr;71(2):97-101.

31. Bremer BA, Wert KM, Durica AL, Weaver A. Neuropsychological, physical, and psychosocial functioning of individuals with end-stage renal disease. Ann Behav Med. 1997;19(4):348-52.

32. Nussbaum J, Garcia RK. Restorative physical and occupational therapy: a critical need for patients with chronic kidney and end-stage renal disease. Adv Chronic Kidney Dis. 2009 Nov;16(6):529-35.

33. Roshanravan B, Gamboa J, Wilund K. Exercise and CKD: skeletal muscle dysfunction and practical application of exercise to prevent and treat physical impairments in CKD. Am J Kidney Dis. 2017 Jun;69(6):837-52.

34. Greenwood SA, Koufaki P, Rush R, Macdougall IC, Mercer TH, British Renal Society Rehabilitation Network. Exercise counselling practices for patients with chronic kidney disease in the UK: a renal multidisciplinary team perspective. Nephron Clin Pract. 2014 Oct 29;128(1-2):67-72. 
35. Greenwood SA, Lindup H, Taylor K, Koufaki P, Rush R, Macdougall IC, et al. Evaluation of a pragmatic exercise rehabilitation programme in chronic kidney disease. Nephrol Dial Transplant. 2012 Oct;27 Suppl 3:iii126-34.

36. Corsonello A, Fusco S, Bustacchini S, Chiatti C, Moresi R, Bonfigli AR, et al. Special considerations for the treatment of chronic kidney disease in the elderly. Expert Rev Clin Pharmacol. 2016 Mar 4;9(5):727-37.

37. Cheng XS, Myers JN, Chertow GM, Rabkin R, Chan KN, Chen Y, et al. Prehabilitation for kidney transplant candidates: Is it time? Clin Transplant. 2017 Jul 10;31(8). 